\title{
Comunicação
}

[Communication]

\section{Imunogenicidade da proteína $M$ recombinante de Streptococcus equi subsp. equi coadministrada com um adjuvante molecular}

\author{
[Immunogenicity of recombinant $M$ protein of Streptococcus equi subsp. equi co administered \\ with a molecular adjuvant] \\ L.F. Maciel ${ }^{1}$, C.G. Magalhães ${ }^{1}$, C.M. Moraes ${ }^{2}$, M.C. Rosa ${ }^{1}$, M. Mendonça ${ }^{3}$, \\ A.N. Moreira ${ }^{1}$, L.A. Dummer ${ }^{1}$, P.F. Finger ${ }^{1}$, M.R.A. Ferreira ${ }^{1}$, \\ F.P.L. Leite ${ }^{1}$, F.R. Conceição ${ }^{1 *}$ \\ ${ }^{1}$ Universidade Federal de Pelotas - Pelotas, RS \\ ${ }^{2}$ Universidade Federal do Pará - Instituto de Medicina Veterinária - Faculdade de Veterinária - Castanhal, PA \\ ${ }^{3}$ Universidade Federal Rural de Pernambuco - Garanhuns, PE
}

O Brasil possui o maior rebanho de equinos da América Latina e o terceiro maior do mundo. $\mathrm{O}$ agronegócio relacionado à criação de cavalos vem crescendo no cenário nacional e é responsável pela geração de cerca de três milhões de empregos (Brasil, 2016). A criação de animais de alta qualidade eleva a perspectiva de crescimento do setor, porém a ocorrência de doenças infecciosas pode contrapor essa tendência.

A adenite equina, causada por Streptococcus equi subsp. equi, é uma das enfermidades mais relevantes entre aquelas que acometem o trato respiratório de equinos e, com isso, a busca por vacinas mais eficientes é importante, uma vez que aquelas atualmente disponíveis no Brasil apresentam eficácia de apenas 50\% (Libardoni, 2015). A vacinação contra S. equi é recomendada em instalações onde a doença é um problema endêmico ou em animais com risco de exposição ao patógeno. É uma doença distribuída de forma uniforme em todas as regiões do estado do Rio Grande do Sul (RS), terceiro maior rebanho do país, sendo prevalente em 5,86\% dos rebanhos (Libardoni et al., 2016).

Considerando que o uso de adjuvantes é importante para aumentar, modular e/ou prolongar a resposta imune, a subunidade B da enterotoxina termolábil de Escherichia coli (LTB), atóxica, vem se destacando

Recebido em 3 de janeiro de 2017

Aceito em 19 de janeiro de 2017

*Autor para correspondência (corresponding author)

E-mail: fabricio.rochedo@ufpel.edu.br principalmente como adjuvante molecular da imunidade de mucosa (imunidade local), potencializando a resposta imune humoral e celular antígeno-específica (Fischer et al., 2010; Hora et al., 2011). Entretanto, a sua avaliação como adjuvante molecular parenteral (imunidade sistêmica) ainda foi pouco explorada. Assim, o objetivo deste trabalho foi avaliar, em camundongos e equinos, a imunogenicidade da proteína $\mathrm{M}$ de $S$. equi subsp. equi (SeM) coadministrada com a LTB, sendo ambas as proteínas produzidas por meio da tecnologia do DNA recombinante.

A expressão da LTB recombinante (rLTB) foi realizada segundo Fischer et al. (2010). Para expressar a SeM recombinante (rSeM), a sequência codificadora da SeM de $S$. equi (GenBank U73162) foi amplificada por PCR, utilizando-se os oligonucleotídeos: 5'CGGGATCCTCGAGGTTAGTCGTAC3' (forward) e 5'GGGGTACCTTAGTTTTCTTTGCGT3' (reverse) e clonada no vetor de expressão pAE, gerando o vetor $\mathrm{pAE} / \mathrm{sem}$, utilizado para transformar $E$. coli Top 10 competente (Invitrogen, EUA). A seleção dos transformantes foi realizada em meio LuriaBertani (LB - 1\% triptona, $0,5 \%$ extrato de levedura, $1 \% \mathrm{NaCl}$ ) com $100 \mu \mathrm{g} / \mathrm{mL}$ de ampicilina (Sigma-Aldrich, EUA). 
Os clones $\mathrm{pAE} / \mathrm{sem}$ e $\mathrm{pAE} / \mathrm{lt}$ b foram usados para transformar por choque térmico a cepa de expressão E. coli BL21 (DE3) Star $^{\mathrm{TM}}$ (Invitrogen, EUA) e foram cultivados em meio LB com $100 \mu \mathrm{g} / \mathrm{mL}$ de ampicilina. Logo que o cultivo atingiu a densidade óptica $\left(\mathrm{DO}_{600}\right)$ de 0,8 , a expressão foi induzida com $0,5 \mathrm{mM}$ de isopropil $\beta$-D-tiogalactopiranosídeo (IPTG) por três horas a $37^{\circ} \mathrm{C}$. A solubilização dos corpos de inclusão de rSeM foi realizada em tampão contendo $\mathrm{N}$ Lauroylsarcosine $(100 \mathrm{mM}$ Tris, $200 \mathrm{mM} \mathrm{NaCl}$, $0,2 \%$ de N-Lauroylsarcosine, $\mathrm{pH} 8,0)$ e de rLTB, em tampão contendo ureia $(100 \mathrm{mM}$ Tris, $200 \mathrm{mM} \mathrm{NaCl}, 6 \mathrm{M}$ de ureia, $\mathrm{pH} 8,0)$. As proteínas recombinantes foram dialisadas contra o mesmo tampão, porém sem os agentes desnaturantes. Ambas as proteínas foram concentradas com polietilenoglicol (PEG) e quantificadas pelo método de Bradford. A caracterização de rSeM e rLTB foi realizada por eletroforese em gel desnaturante de poliacrilamida (SDS-PAGE) $12 \%$ e Western blot (WB) com anticorpo monoclonal (MAb) anti6xHis (Sigma-Aldrich, EUA).

Para a avaliação da imunogenicidade, foram utilizados 72 camundongos Balb/c, de seis a oito semanas, fêmeas, divididos em oito grupos. Os animais foram inoculados nos dias zero, 14 e 21, por via intramuscular (IM) ou intranasal (IN) (aplicação com micropipeta) (Tab. 1). Amostras de sangue foram coletadas por punção do plexo venoso retro-ocular nos dias zero, sete, 14, 21 e 42 , para obtenção de soro, e conservadas a $-20^{\circ} \mathrm{C}$ até a utilização.

Os níveis séricos (dias zero, sete, 14, 21 e 42) e a titulação de imunoglobulinas (Igs) séricas antirSeM de camundongos (dia 42) foram realizados mediante ELISA indireto. Placas de 96 cavidades (Cralplast, Brasil) foram sensibilizadas com $12 \mu \mathrm{g} / \mathrm{mL}$ de rSeM diluída em tampão carbonatobicarbonato $\mathrm{pH} 9,6$ (TCB) e mantidas a $4^{\circ} \mathrm{C}$ por 18h. Para avaliação dos níveis séricos de Igs séricas anti-rSeM, os soros foram diluídos de 1:200. Já para a titulação de Igs séricas anti$\mathrm{rSeM}$, os soros foram diluídos de 1:200 a 1:145800 para os grupos inoculados por via IM e de 1:40 a 1:320 para os grupos inoculados por via IN. Anti-Igs totais de camundongo conjugadas com peroxidase (Sigma-Aldrich, EUA) foram acrescentadas na diluição 1:4000. A reação foi revelada com solução cromógena (ortofenilenodiamina - OPD; 0,2M tampão citrato-fosfato $\mathrm{pH} 4,0 ; 0,01 \%$ de $\mathrm{H}_{2} \mathrm{O}_{2}$ ), e a leitura da DO realizada a $450 \mathrm{~nm}\left(\mathrm{DO}_{450}\right)$, após $15 \mathrm{~min}$ da adição do revelador. Cada etapa foi incubada a $37^{\circ} \mathrm{C}$ por $1,5 \mathrm{~h}$ e, entre cada uma delas, foram realizadas três lavagens com PBS + Tween $0,05 \%(\mathrm{v} / \mathrm{v})(\mathrm{PBS}-\mathrm{T})$.

Tabela 1. Formulações vacinais e doses utilizadas para inocular camundongos

\begin{tabular}{ccccc}
\hline \multirow{2}{*}{ Grupo } & $\begin{array}{c}\text { Via de } \\
\text { administração }\end{array}$ & Formulações vacinais & Dose & $n^{*}$ \\
\hline 1 & $\mathrm{IM}$ & $\mathrm{rSeM}+\mathrm{Al}(\mathrm{OH})_{3}$ & $25 \mu \mathrm{g}+10 \%$ & 9 \\
2 & $\mathrm{IM}$ & $\mathrm{rSeM}+\mathrm{rLTB}$ & $25 \mu \mathrm{g}+10 \mu \mathrm{g}$ & 9 \\
3 & $\mathrm{IM}$ & $\mathrm{rSeM}+\mathrm{rLTB}+\mathrm{Al}(\mathrm{OH})_{3}$ & $25 \mu \mathrm{g}+10 \mu \mathrm{g}+10 \%$ & 9 \\
4 & $\mathrm{IM}$ & $\mathrm{rLTB}+\mathrm{Al}(\mathrm{OH})_{3}$ & $10 \mu \mathrm{g}+10 \%$ & 9 \\
5 & $\mathrm{IM}$ & $\mathrm{rSeM}$ & $25 \mu \mathrm{g}$ & 9 \\
6 & $\mathrm{IN}$ & $\mathrm{rSeM}$ & $25 \mu \mathrm{g}$ & 9 \\
7 & $\mathrm{IN}$ & $\mathrm{rSeM}+\mathrm{rLTB}$ & $25 \mu \mathrm{g}+10 \mu \mathrm{g}$ & 9 \\
8 & $\mathrm{IN}$ & $\mathrm{rLTB}$ & $10 \mu \mathrm{g}$ & 9 \\
\hline
\end{tabular}

${ }^{*} n=$ número de animais por grupo.

Equinos com baixos títulos de Igs séricas antirSeM foram selecionados para avaliação das formulações vacinais. Com isso, 28 animais, de um a 19 anos de idade, sem histórico de garrotilho, foram distribuídos em sete grupos. A inoculação dos cavalos foi realizada nos dias zero e 28, por via IM ou IN (aplicação com spray) (Tab. 2). O sangue foi coletado, por punção da veia jugular, nos dias zero, 28 e 42. Secreção nasal dos equinos foi coletada como previamente descrito por Nelson et al. (1998). Também foram coletados swabs da língua dos cavalos em uma área padronizada por este grupo de $9 \mathrm{~cm}^{2}(3 \mathrm{~cm} \times 3 \mathrm{~cm})$. O conteúdo nasal e o da língua foram imersos em $5 \mathrm{~mL}$ de PBS contendo $5 \mathrm{mM}$ de EDTA, $1 \mathrm{mM}$ de PMSF, $0,5 \%$ de gelatina, $0,05 \%$ de Tween 80 e $0,05 \%$ de $\mathrm{NaN}_{3}$, homogeneizados em vórtex para liberação do material, centrifugados em $13.000 \mathrm{rpm}$ por $10 \mathrm{~min}$ e o sobrenadante estocado a $-20^{\circ} \mathrm{C}$ até o uso. 
Os títulos de Igs anti-rSeM no soro dos cavalos foram determinados por meio de ELISA indireto com amostras coletadas do dia 42. Para tal, as placas foram sensibilizadas com $100 \mu \mathrm{L}$ /poço de rSeM $(12 \mu \mathrm{g} / \mathrm{mL})$ diluída em TCB e mantidas a $4^{\circ} \mathrm{C}$ por $18 \mathrm{~h}$, depois foram bloqueadas com leite em pó 5\% (Molico; p/v). Para a titulação, foram feitas diluições de 1:1000 a 1:16000. Em seguida, anti-IgG de cavalo conjugado à peroxidase (Sigma-Aldrich, EUA) foi adicionado na diluição 1:6000. A reação foi revelada usando-se solução cromógena e avaliada a $\mathrm{DO}_{450}$ após 15 minutos. Cada etapa foi incubada a $37^{\circ} \mathrm{C}$ por $1,5 \mathrm{~h}$ e entre cada uma delas foram realizadas três lavagens com PBS-T.

Para avaliação de IgA secretora anti-rSeM, $200 \mu \mathrm{L}$ de amostra foram aplicados diretamente na placa previamente sensibilizada com rSeM, e incubada em temperatura ambiente por $18 \mathrm{~h}$. Após, foi acrescentado anti-IgA de cavalo conjugado à peroxidase (Bethyl Laboratories, EUA), na diluição $1: 2000$ por duas horas a $37^{\circ} \mathrm{C}$. A reação foi revelada usando-se solução cromógena por 15 minutos, e foi feita leitura na $\mathrm{DO}_{450}$.

Tabela 2. Formulações vacinais e doses utilizadas para inocular cavalos

\begin{tabular}{cccccc}
\hline Grupo & $\begin{array}{c}\text { Via de } \\
\text { administração }\end{array}$ & Forumulação & Dose & $*_{n}$ & Idade \\
\hline 1 & IN & PBS & $2 \mathrm{~mL}$ & 4 & $1,3,4,6$ \\
2 & $\mathrm{IN}$ & $\mathrm{rSeM}$ & $100 \mu \mathrm{g}$ & 4 & $1^{\#}, 3,4,9$ \\
3 & $\mathrm{IN}$ & $\mathrm{rSeM}+\mathrm{rLTB}$ & $100 \mu \mathrm{g}+50 \mu \mathrm{g}$ & 4 & $1^{\#}, 3,4^{\#}, 11$ \\
4 & $\mathrm{IM}$ & $\mathrm{PBS}$ & $2 \mathrm{~mL}$ & 4 & $3,3,4,6$ \\
5 & $\mathrm{IM}$ & $\mathrm{rSeM}$ & $100 \mu \mathrm{g}$ & 4 & $1^{\#}, 3,4^{\#}, 19$ \\
6 & $\mathrm{IM}$ & $\mathrm{rSeM}+\mathrm{rLTB}$ & $100 \mu \mathrm{g}+50 \mu \mathrm{g}$ & 4 & $3,3,4,9$ \\
7 & $\mathrm{IM}$ & $\mathrm{rSeM}+$ & $100 \mu \mathrm{g}+15 \%$ & 4 & $2,3^{\#}, 9,19$ \\
\hline
\end{tabular}

*n= número de animais por grupo.

\#Animais desenvolveram garrotilho durante o experimento e foram excluídos das análises.

A análise estatística foi realizada por meio do teste T Students, no software GraphPad Prism 5, para determinar as diferenças significativas $(\mathrm{P}<0,05)$ entre médias aritméticas.

Este trabalho foi aprovado pelo Comitê de Ética em Experimentação Animal (Ceea) da Universidade Federal de Pelotas (protocolo $\mathrm{n}^{\circ}$ 2354-2014). Os animais foram mantidos $\mathrm{e}$ manuseados de acordo com os requisitos legais previstos na Lei Nacional de Proteção aos Animais de Experimentação (Brasil, 2008).

A amplificação da sequência codificadora da proteína $\mathrm{SeM}$ resultou em um fragmento de $1600 \mathrm{pb}$, conforme esperado. A clonagem no vetor de expressão pAE foi caracterizada por restrição enzimática e por PCR (dados não mostrados). As proteínas rSeM e rLTB foram reconhecidas por meio de WB, utilizando-se anticorpo anti-6xHis (dados não mostrados). A expressão de rSeM em E. coli gerou uma proteína com massa molecular aparente de $58 \mathrm{kDa}$, o que confirma os resultados descritos por Galáne e Timoney (1987), e a expressão de rLTB, uma proteína de aproximadamente $14 \mathrm{kDa}$ (Fischer et al., 2010).

$\mathrm{Na}$ avaliação dos níveis séricos de Igs anti-rSeM nos diferentes dias, os camundongos inoculados por via IN não apresentaram diferença em relação ao controle, assim como aos animais inoculados por via IM. Todos os animais dos grupos inoculados por via IM com diferentes formulações contendo rSeM apresentaram incremento nos níveis de Igs anti-rSeM sete dias após a primeira dose, em comparação ao grupo controle, e se mantiveram até os 42 dias (Fig. 1A e 1C). Foi observado um aumento significativo de pelo menos 14 vezes no título médio de Igs totais anti-rSeM (dia 42) induzidas pelas formulações vacinais contendo $\mathrm{rSeM}$ em relação ao controle $\left(\mathrm{rLTB}+\mathrm{Al}(\mathrm{OH})_{3}\right)$, no soro dos camundongos inoculados via IM. Nos camundongos inoculados via IN, o grupo rSeM + rLTB apresentou maior título médio de Igs, porém a diferença entre os grupos não foi significativa (Fig. 1B). 
A

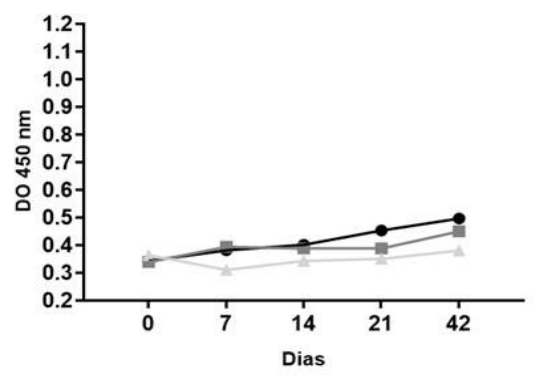

C

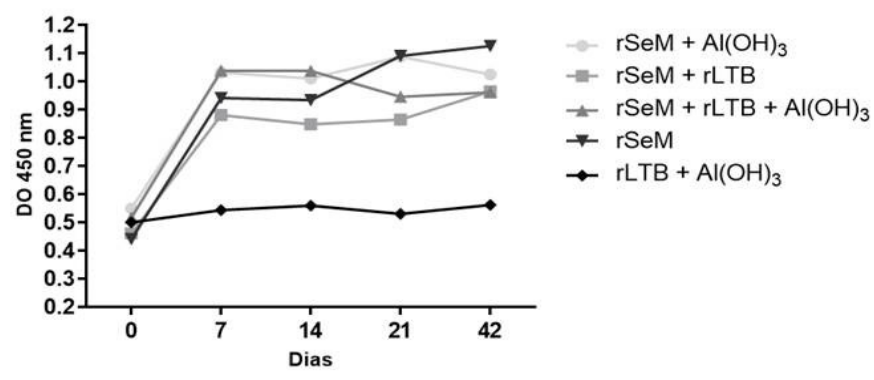

B

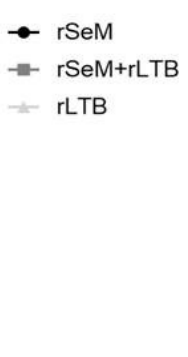

-rSeM+rLTB

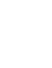


O presente estudo mostrou que a rSeM é bastante imunogênica, como descrito por Moraes (2008), uma vez que camundongos e cavalos tiveram aumento nos títulos de Igs séricas anti-rSeM, até mesmo quando o antígeno recombinante foi administrado sem adjuvante. A formulação rSeM + rLTB foi o que estimulou o maior título de Igs séricas anti-rSeM em ambas as espécies, independentemente da via de administração, demonstrando o papel adjuvante da rLTB, o que corrobora dados obtidos em estudos anteriores (Conceição et al., 2006; Fischer et al., 2010; Hora et al., 2011). É importante observar que a rLTB gerou títulos semelhantes de anticorpos em comparação com o adjuvante comumente utilizado em vacinas comerciais, o $\mathrm{Al}(\mathrm{OH})_{3}$, podendo ser uma alternativa viável para utilização em formulações vacinais de interesse veterinário. Entretanto, a rLTB não mostrou sinergismo com o $\mathrm{Al}(\mathrm{OH})_{3}$, visto que os camundongos vacinados com essa combinação apresentaram título médio de Igs séricas antirSeM levemente inferior aos demais tratamentos.

A imunidade ante a infecção por $S$. equi pode ser presumida por meio da avaliação do título de anticorpos anti-SeM por ELISA indireto, sendo considerados elevados, na maioria dos cavalos, títulos superiores a 1:1600 e, provavelmente, capazes de conferir imunidade à doença (Mallicote, 2015). Os resultados aqui apresentados demonstram um título superior a 1:5000 nos cavalos inoculados por via IM, sugerindo uma possível proteção no tocante à infecção por S. equi.
Neste estudo, não foi detectado um aumento nos níveis de $\operatorname{IgA}$ secretora anti-rSeM, talvez porque os cavalos tenham sido inoculados com apenas duas doses de $100 \mu \mathrm{g}$ de rSeM. Sheoran et al. (2002) conseguiram induzir resposta imune de mucosa específica em cavalos usando três doses, sendo a primeira vacinação com $500 \mu \mathrm{g}$ e as demais com $300 \mu \mathrm{g}$ de uma fração da proteína SeM fusionada à subunidade $\mathrm{B}$ da toxina colérica, que apresenta mais de $80 \%$ de homologia com a LTB (Simmons et al., 2001). Flock et al. (2004), ao utilizarem outros antígenos de $S$. equi com LTB, obtiveram maiores títulos de $\operatorname{IgA}$ na mucosa nasal de cavalos por meio da combinação de vias subcutânea e intranasal. Isso sugere que talvez a combinação de vias de administração e/ou ainda $\mathrm{o}$ aumento no número de doses vacinais $\mathrm{e}$ concentração de antígeno em cada dose poderiam estar envolvidos numa melhor estimulação da imunidade de mucosa.

Os resultados obtidos neste trabalho demonstram a potencial utilização de $\mathrm{rSeM}$ como antígeno vacinal e da rLTB como adjuvante molecular, tendo em vista que a formulação foi imunogênica e inócua para camundongos e cavalos. Porém, mais estudos devem ser realizados para determinar a concentração de antígeno e o número de doses adequadas, assim como para avaliar a eficácia dessa vacina a campo.

Palavras-chave: Garrotilho, Streptococcus equi, rSeM e rLTB

\begin{abstract}
The strangles is an infectious disease that affects horses from all ages and causes important economic losses in the equine-related business. The aim of this work was to evaluate the immunogenicity of the recombinant $M$ protein from Streptococcus equi ( $r S e M)$ co-administered with the recombinant heat-labile enterotoxin B subunit from Escherichia coli ( $r L T B)$ in mice and horses. A total of 72 female Balb-c mice were divided into eight groups and 18 horses were divided into six groups. The animals were inoculated by intramuscular (IM) or intranasal (IN) routes with different treatments of rSeM, rLTB and/or $\mathrm{Al}(\mathrm{OH})_{3}$. The results obtained in both species, independent of administration routes, demonstrated that $r S e M+$ rLTB had higher levels of specific serum immunoglobulins, however, in mucosal immunity the increase was not identified. Thus, the use of rSeM as vaccine antigen and rLTB as adjuvant can be a potential tool in the control of equine strangles.
\end{abstract}

Keyword: Strangles, Streptococcus equi, rSeM and rLTB 


\section{REFERÊNCIAS}

BRASIL. Conselho Nacional de Controle de Experimentação Animal. Lei Nacional de Proteção aos Animais de Experimentação. Lei ${ }^{\circ}$ 11.794. Diário Oficial, Brasília, 8 out. 2008.

BRASIL. Ministério da Agricultura pecuária e desenvolvimento. Lei $\mathrm{N}^{\circ} 11.794$, de 8 de outubro de 2008. Disponível em: $<$ http://www.agricultura.gov.br/animal/especies/ equideos $>$. Acessado em 15 jan. 2016.

CONCEIÇÃO, F.R.; MOREIRA, A.N.; DELLAGOSTIN, O.A. A recombinant chimera composed of R1 repeat region of Mycoplasma hyopneumoniae P97 adhesin with Escherichia coli heat-labile enterotoxin B subunit elicits immune response in mice. Vaccine, v.24, p.5734$5743,2006$.

FISCHER, G.; CONCEICAO, F.R.; LEITE, F.P.L. et al. Recombinant Escherichia coli heatlabile enterotoxin B subunit humoral adjuvant effect depends on dose and administration route. World J. Microbiol. Biotechnol., v.26, p.489-95, 2010.

FLOCK, M.; JACOBSSON, K.; FRYKBERG, L. et al. Recombinant Streptococcus equi proteins protect mice in challenge experiments and induce immune response in horses. Infect. Immun., v.72, p.3228-3236, 2004.

GALÁN, J.E.; TIMONEY, J.F. Molecular analysis of the M protein of Streptococcus equi and cloning and expression of the $\mathrm{M}$ protein gene in Escherichia coli. Infect. Immun., v.55, p.31813187, 1987.

HORA, V.P.; CONCEIÇÃO, F.R.; DELLAGOSTIN, O.A.; DOOLAN, D.L. Nontoxic derivatives of LT as potent adjuvants. Vaccine, v.29, p.1538-1544, 2011.
LIBARDONI, F. Equinos portadores de Streptococcus equi subespécie equi: Prevalência, fatores de risco e caracterização de alelos seM. 2015. 58f. Tese (Pós-Graduação em Medicina Veterinária), Universidade Federal de Santa Maria, Santa Maria, RS.

LIBARDONI, F.; MACHADO, G.; GRESSLER, L.T. et al. Prevalence of Streptococcus equi subsp. equi in horses and associated risk factors in the state of Rio Grande do Sul, Brazil. Res. Vet. Sci., v.104, p.53-57, 2016.

MALLICOTE, M. Update on Streptococcus equi subsp equi infections. Vet. Clin. N. Am. Equine Pract., v.31, p.27-41, 2015.

MORAES, C.M. Produção e avaliação de proteína SeM recombinante para o controle de Adenite Equina. 2008. 79f. Tese (Doutorado em Biotecnologia) - Centro de Biotecnologia, Universidade Federal de Pelotas, RS.

NELSON, K.M.; SCHRAM, B.R.; MCGREGOR, M.W. et al. Local and systemic isotype-specific antibody responses to equine influenza virus infection versus conventional vaccination. Vaccine, v.16, p.1306-1313, 1998.

SHEORAN, A.S.; ARTIUSHIN, S.; TIMONEY, J.F. Nasal mucosal immunogenicity for the horse of a SeM peptide of Streptococcus equi genetically coupled to cholera toxin. Vaccine, v.20, p.1653-1659, 2002.

SIMMONS, C.P.; GHAEM-MAGAMI, M.; PETROVSKA, L. et al. Immunomodulation using bacterial enterotoxins. Scand. J. Immunol., v.53, p.218-26, 2001. 